EPJ Web of Conferences 20, 03003 (2012)

DOI: $10.1051 /$ epjconf/20122003003

(C) Owned by the authors, published by EDP Sciences, 2012

\title{
SU(2) color NJL model and EOS of quark-hadron matter at finite temperature and density
}

\author{
Shotaro Imai ${ }^{1, \text { a }}$, Hiroshi Toki ${ }^{1}$, and Wolfram Weise ${ }^{2}$ \\ 1 Research Center for Nuclear Physics, Osaka University, Ibaraki, Osaka 567-0047, Japan \\ 2 Physik-Department, Technische Universität München, D-85747 Garching, Germany
}

\begin{abstract}
We study the NJL model with the Polyakov loop in the SU(2)-color case for the EOS of quark-hadron matter at finite temperature and density. We consider the spontaneous chiral symmetry breaking and the diquark condensation together with the behavior of the Polyakov loop for the phase diagram of quark-hadron matter. We discuss the spectrum of mesons and diquark baryons (boson) at finite temperature and density. We derive also the linear sigma model Lagrangian for diquark baryon and mesons.
\end{abstract}

Key words. Two-color - NJL model - Polyakov loop potential - Chiral condensation Diquark condensation - Temperature and density

\section{Introduction}

The quarks and gluons are the ingredients of the quantum chromodynamics (QCD). At low temperature and density, the quarks and gluons are confined in the form of hadrons as colorless particles. However, at high temperature and density, the quarks and gluons are de-confined and become explicit degrees of freedom in this circumstance. Hence, for the discussion of strongly interacting matter in various situations, we ought to describe the dynamics of both the quarks and gluons and at the same time the baryons and mesons.

We often use the Nambu-Jona-Lasinio (NJL) model as an effective theory of strongly interacting matter, where the chiral symmetry and its spontaneous breaking are incorporated [1]. The NJL model is further armed by introducing the Polyakov loop to simulate the effect of color confinement [2-4]. This model, called as PNJL model, is by now used very often for the discussion of quark-hadron matter at finite temperature and density. The PNJL model results can be compared with lattice QCD results very well at finite temperature with zero density. As for finite density, the PNJL model is able to provide equations of state and phases of quark-hadron matter at various densities and temperatures.

Recently there are some studies of quark-hadron matter at finite temperature and density with the lattice QCD (LQCD) simulation for the case of two color [5,6]. In this case, there is no sign problem and the LQCD simulation is able to provide equations of state of two color quark-hadron matter at finite temperature and density. There is a theoretical study to understand the behavior of various observables by using the symmetry argument [7]. It is then very interesting to work out the PNJL model for the case of the two color and compare with the LQCD simulation results. In the PNJL model we are able to provide the meson spectrum and di-quark baryon spectrum at various temperature and densities. A pioneering study in the NJL mode was performed by Ratti and Weise [8]. Introducing the Polyakov loop potential, we are able to study the role of confinement and further study in detail the hadron spectrum and also equations of state at various temperatures and densities.

\footnotetext{
a e-mail: imais@rcnp.osaka-u.ac.jp
}

This is an Open Access article distributed under the terms of the Creative Commons Attribution-Noncommercial License 3.0, which permits unrestricted use, distribution, and reproduction in any noncommercial medium, provided the original work is properly cited. 


\section{EPJ Web of Conferences}

\section{NJL model with Polyakov loop}

We want to work out the NJL model with Polyakov loop (PNJL model) for the two-color case to obtain an effective lagrangian with mesons and diquark-baryons in both chiral and diquark condensed phases. The PNJL Lagrangian with diquark channnel is

$$
\begin{aligned}
\mathcal{L} & =\bar{\psi}\left(i \gamma_{\mu} D^{\mu}-m_{0}+\gamma_{0} \mu\right) \psi+\frac{G_{0}}{2}\left[(\bar{\psi} \psi)^{2}+\left(\bar{\psi} i \gamma_{5} \tau \psi\right)^{2}\right] \\
& +\frac{H_{0}}{2}\left(\bar{\psi} i \gamma_{5} t_{2} \tau_{2} C \bar{\psi}^{T}\right)\left(\psi^{T} C i \gamma_{5} t_{2} \tau_{2} \psi\right)-U\left(\Phi[A], \Phi^{*}[A] ; T\right) .
\end{aligned}
$$

The covariant derivative is $D^{\mu}=\partial^{\mu}-i A^{\mu}$ and the Polakov loop potential is $U\left(\Phi[A], \Phi^{*}[A] ; T\right)$. In the Polyakov gauge the background color gauge field is diagonal in the color space and is defined as $A_{4}=i A_{0}$ with the gauge field $A^{\mu}=g A_{a}^{\mu} \frac{t_{a}}{2}$ with $t_{a}$ being the $S U(2)$ Pauli matrix operator in the color space. We take two flavors for the quark $\psi$ with the isospin operator $\tau_{a}$ and their masses are set equal as $m_{u}=m_{d}=m_{0}=5.4 \mathrm{MeV} . \psi^{T}$ denotes the transpose of $\psi$. The charge conjugation operator for fermions $C=i \gamma_{0} \gamma_{2}$ have been introduced. The coupling constant $G_{0}$ for the meson channel is equal to the coupling constant $H_{0}$ for the diqaurk channel as well-known as Pauli-Güersey symmetry which charactrize the two-color QCD, hence the order parameters, the chiral condensate $\langle\bar{\psi} \psi\rangle$ and the diquark condensate $\langle\psi \psi\rangle$, are equivalent in the chiral limit $\left(m_{0}=0\right)$ with zero chemical potential [8].

The effective Polyakov loop potential $U$ is written in terms of the traced Polyakov loop

$$
\Phi=\frac{1}{N_{c}} \operatorname{tr}_{c} \mathcal{P} \exp \left[i \int_{0}^{\beta} d \tau A_{4}(\mathbf{x}, \tau)\right] .
$$

In the two-color case, the traced Polyakov loop is real,$\Phi^{*}=\Phi$. With the traced Polyakov loop, we can express the potential as [9]

$$
U(\Phi, T)=-b T\left(24 \Phi^{2} e^{-\beta a}+\ln \left(1-\Phi^{2}\right)\right),
$$

which comes from the Haar measure in $S U(2)$. The two parameters $a=858.1 \mathrm{MeV}$ and $b^{1 / 3}=210.5$ $\mathrm{MeV}$ are taken from the work of Brauner et al. [9] so that we can make a coherent discussion with previous works.

To proceed, we use the bosonization technique and write the Lagrangian in terms of auxiliary fields [10]. We introduce meson and diquark auxiliary fields as $\sigma, \pi, \Delta$ and $\Delta^{*}$. The partition function of the PNJL model including auxiliary fields is written as

$$
Z=\int D \bar{\psi} D \psi D \sigma D \pi D \Delta D \Delta^{*} \exp \left(i \int d^{4} x \mathcal{L}_{a u x}\right),
$$

and the Lagrangian is written as

$$
\mathcal{L}_{a u x}=\frac{1}{2}\left(\bar{\psi} \psi^{T} C\right)\left(\begin{array}{cc}
S^{-1}(\mu) & g_{d} \gamma_{5} t_{2} \tau_{2} \Delta \\
-g_{d} \Delta^{*} \gamma_{5} t_{2} \tau_{2} & S^{-1}(-\mu)
\end{array}\right)\left(\begin{array}{c}
\psi \\
C \bar{\psi}^{T}
\end{array}\right)-\frac{1}{2} \delta \mu_{0}^{2}\left(\sigma^{2}+\pi^{2}\right)-\frac{1}{2} M_{d}^{2} \Delta^{*} \Delta
$$

with $S^{-1}( \pm \mu)=i \gamma_{\mu} \partial^{\mu}-m_{0} \pm \gamma_{0} \mu-g_{0}\left(\sigma \pm i \gamma_{5} \pi \cdot \tau\right)$. We have introduced a meson coupling constant $g_{0}$ and a diquark coupling constant $g_{d}$ together with bare mass terms $\delta \mu_{0}$ and $M_{d}$ with the relations with the meson and diquark coupling constants as $\frac{g_{0}^{2}}{\delta \mu_{0}^{2}}=G_{0}$ and $\frac{g_{d}^{2}}{M_{d}^{2}}=H_{0}$. Here, we have dropped writing the Polyakov loop potential. After introducing the chiral condensate $\sigma(x) \rightarrow \sigma_{0}+s(x)$ and the diquark condensate $\Delta(x) \rightarrow \Delta_{0}+d(x), \Delta^{*}(x) \rightarrow \Delta_{0}^{*}+d^{*}(x)$ at the mean field level, we can integrate out the Lagrangian over the quark fields. The result is written as

$$
\begin{aligned}
\mathcal{L}_{e f f} & =-\frac{i}{2} \operatorname{tr}\left(\ln \hat{S}^{-1}+\ln (1+\hat{S} \hat{K})\right)-\frac{1}{2} \delta \mu_{0}^{2} \sigma_{0}^{2}-\frac{1}{2} \delta \mu_{0}^{2}\left(s^{2}+\pi^{2}\right)-\delta \mu_{0}^{2} \sigma_{0} s \\
& -\frac{1}{2} M_{d}^{2} \Delta_{0}^{*} \Delta_{0}-\frac{1}{2} M_{d}^{2}\left(\Delta_{0}^{*} d+d^{*} \Delta_{0}\right)-\frac{1}{2} M_{d}^{2} d^{*} d .
\end{aligned}
$$


The matrices $\hat{S}^{-1}$ and $\hat{K}$ are defined as,

$$
\hat{S}^{-1}=\left(\begin{array}{cc}
S_{0}^{-1}(\mu) & \Delta^{-} \\
\Delta^{+} & S_{0}^{-1}(-\mu)
\end{array}\right), \quad \hat{K}=\left(\begin{array}{cc}
-g_{0}\left(s+i \gamma_{5} \pi \cdot \tau\right) & g_{d} \gamma_{5} t_{2} \tau_{2} d \\
-g_{d} d^{*} \gamma_{5} t_{2} \tau_{2} & -g_{0}\left(s-i \gamma_{5} \pi \cdot \tau\right)
\end{array}\right)
$$

Here, $\Delta^{-}=g_{d} \gamma_{5} t_{2} \tau_{2} \Delta_{0}, \Delta^{+}=-g_{d} \Delta_{0}^{*} \gamma_{5} t_{2} \tau_{2}$ and $S_{0}^{-1}( \pm \mu)=i \gamma_{\mu} \partial^{\mu}-m \pm \gamma_{0} \mu$ are used. The constituent quark mass have been defined as $m=m_{0}+g_{0} \sigma_{0}$. We introduce projection operators to handle quark propagators as follows [11]. The Nambu-Gorkov propagator $\hat{S}$ is determined by solving $\hat{S} \hat{S}^{-1}=1$ as

$$
\hat{S}=\left(\begin{array}{ll}
G^{+} & H^{-} \\
H^{+} & G^{-}
\end{array}\right)
$$

with the components

$$
G^{ \pm}=\frac{p_{0} \pm E_{p}^{-}}{p_{0}^{2}-E_{\Delta}^{-2}} \Lambda_{ \pm} \gamma_{0}+\frac{p_{0} \mp E_{p}^{+}}{p_{0}^{2}-E_{\Delta}^{+2}} \Lambda_{\mp} \gamma_{0}, \quad H^{ \pm}=\frac{\Delta^{ \pm}}{p_{0}^{2}-E_{\Delta}^{ \pm 2}} \tilde{\Lambda}_{+}+\frac{\Delta^{ \pm}}{p_{0}^{2}-E_{\Delta}^{\mp 2}} \tilde{\Lambda}_{-},
$$

where $E_{p}^{ \pm}=E_{p} \pm \mu$ with $E_{p}=\sqrt{m^{2}+\mathbf{p}^{2}}$ and $E_{\Delta}^{ \pm 2}=E_{p}^{ \pm 2}+g_{d}^{2}\left|\Delta_{0}\right|^{2}$ have been used.

\section{The mean field Lagrangian and thermodynamical potential}

We work out the mean field part of the Lagrangian, which is written as

$$
\mathcal{L}_{M F}=-\frac{i}{2} \operatorname{tr} \ln \hat{S}^{-1}-\frac{1}{2} \delta \mu_{0}^{2} \sigma_{0}^{2}-\frac{1}{2} M_{d}^{2}\left|\Delta_{0}\right|^{2} .
$$

The first term is calculated by using the relation $\operatorname{tr} \ln \hat{S}^{-1}=\ln \operatorname{det} \hat{S}^{-1}$. Here, the determinant is taken over spin, flavor, color and coordinate spaces. We introduce now the temperature using the Matsubara formulation with the replacement $p_{0} \rightarrow i \omega_{n}$ with the Matsubara frequency $\omega_{n}=(2 n+1) \pi T$. We can write the thermodynamical potential as

$$
\Omega=-2 \operatorname{tr} \int \frac{d^{3} p}{(2 \pi)^{3}}\left(\frac{1}{2}\left(E_{\Delta}^{+}+E_{\Delta}^{-}\right)+T\left[\ln \left(1+e^{-\beta E_{\Delta}^{+}}\right)+\ln \left(1+e^{-\beta E_{\Delta}^{-}}\right)\right]\right)+\frac{1}{2} M_{d}^{2} \Delta_{0}^{2}+\frac{\left(m-m_{0}\right)^{2}}{2 G} .
$$

When we have the Polyakov loop effect, we only have to change the log term as $\ln \left(1+e^{-\beta E_{\Delta}^{+}}\right) \rightarrow$ $\frac{1}{2} \ln \left(1+2 \Phi e^{-\beta E_{\Delta}^{+}}+e^{-2 \beta E_{\Delta}^{+}}\right)$with the rule $\Phi^{*} \Phi=1$. The result of the mean field equations are

$$
\begin{aligned}
\frac{\partial \Omega}{\partial m} & =-\operatorname{tr} \int \frac{d^{3} p}{(2 \pi)^{3}} \frac{m}{E_{p}}\left[\frac{E_{p}^{+}}{E_{\Delta}^{+}}\left(1-2 \tilde{f}\left(E_{\Delta}^{+}\right)\right)+\frac{E_{p}^{-}}{E_{\Delta}^{-}}\left(1-2 \tilde{f}\left(E_{\Delta}^{-}\right)\right)\right]+\left(m-m_{0}\right) / G_{0}=0 \\
\frac{\partial \Omega}{\partial \Delta_{0}} & =-\Delta_{0} \operatorname{tr} \int \frac{d^{3} p}{(2 \pi)^{3}}\left[\frac{1}{E_{\Delta}^{+}}\left(1-2 \tilde{f}\left(E_{\Delta}^{+}\right)\right)+\frac{1}{E_{\Delta}^{-}}\left(1-2 \tilde{f}\left(E_{\Delta}^{-}\right)\right)\right]+M_{d}^{2} \Delta_{0}=0
\end{aligned}
$$

where $\tilde{f}(E)$ is the fermi distribution function including Polyakov term

$$
\tilde{f}(E)=\frac{1+\Phi e^{E \beta}}{1+2 \Phi e^{E \beta}+e^{2 E \beta}} .
$$

\section{Hadron Lagrangian with bosonization technique}

We would like to extract the properties of the mesons and diquark-baryons at finite temperature and density. For this purpose, we expand the log term as

$$
-\frac{i}{2} \operatorname{tr} \ln (1+\hat{S} \hat{K})=-\frac{i}{2} \operatorname{tr} \sum_{k=1}^{\infty} \frac{(-1)^{k+1}}{k}(\hat{S} \hat{K})^{k}
$$




\section{EPJ Web of Conferences}

We shall work out the kinetic energies and the mass terms of the mesons and the diquark baryons by working out the $k=2$ terms.

$$
\begin{aligned}
\frac{i}{4} \operatorname{tr}(\hat{S} \hat{K})^{2} & =\int d^{4} x d^{4} y\left\{\Gamma_{s s}(x-y) s(x) s(y)+\Gamma_{p p}(x-y) \pi(x) \cdot \pi(y)+\Gamma_{d d}(x-y) d(x) d(y)+\Gamma_{d^{*} d^{*}}(x-y) d^{*}(x) d^{*}(y)\right. \\
& +\Gamma_{d d^{*}}(x-y) d(x) d^{*}(y)+\Gamma_{d^{*} d}(x-y) d^{*}(x) d(y)+\left(\text { mixing term with } s, d \text { and } d^{*}\right)
\end{aligned}
$$

The propagator terms $\Gamma$ 's are described in Fourier transformed forms as

$$
\Gamma(x-y)=\int \frac{d^{4} q}{(2 \pi)^{4}} e^{i q(x-y)} \Gamma(q) \sim \delta(x-y) \Gamma(0)-i \partial^{\mu} \delta(x-y) \partial_{\mu} \Gamma(0)-\frac{1}{2} \partial^{\mu} \partial^{v} \delta(x-y) \partial_{\mu} \partial_{v} \Gamma(0)
$$

The result of the Fourier transformed propagator for the pion is

$$
\Gamma_{p p}(q)=\frac{i}{4} \operatorname{tr} \int \frac{d^{4} p}{(2 \pi)^{4}} g_{0}^{2}\left(H_{p^{\prime}}^{-} \gamma_{5} H_{p}^{+} \gamma_{5}+H_{p^{\prime}}^{+} \gamma_{5} H_{p}^{-} \gamma_{5}-G_{p^{\prime}}^{+} \gamma_{5} G_{p}^{+} \gamma_{5}-G_{p^{\prime}}^{-} \gamma_{5} G_{p}^{-} \gamma_{5}\right),
$$

which is applied to eq.16. After a lengthy manipulation and repeating sigma propagator $\Gamma_{s s}(q)$ and introduce renormalization program, we get the pion and sigma Lagrangian

$$
\begin{aligned}
& \mathcal{L}_{\pi}=\frac{1}{2} \partial^{\mu} \pi(x) \partial_{\mu} \pi(x)-\frac{1}{2} m_{\pi}^{2} \pi^{2}(x), \\
& \mathcal{L}_{s}=\frac{1}{2} \partial^{\mu} s(x) \partial_{\mu} s(x)-\frac{1}{2} m_{s}^{2} s^{2}(x) .
\end{aligned}
$$

The sigma and pion masses are given as

$$
m_{\pi}^{2}=\left(\frac{1}{G_{0}}-2 I_{2}+4 \mu^{2} I_{0}^{\prime}\right) \frac{1}{I_{0}^{\prime}}, \quad m_{s}^{2}=\left(\frac{1}{G_{0}}-2 I_{2}+4 \mu^{2} I_{0}^{\prime}+4 m^{2} I_{0}\right) \frac{1}{I_{0}^{\prime}}
$$

The divergent integrals $I_{2}, I_{0}$ and $I_{0}^{\prime}$ are defined as

$$
\begin{aligned}
& I_{2}=i \operatorname{tr}_{f c} \int \frac{d^{4} p}{(2 \pi)^{4}}\left(\frac{1}{p_{0}^{2}-E_{\Delta}^{+2}}+\frac{1}{p_{0}^{2}-E_{\Delta}^{-2}}\right), \\
& I_{0}=-i \operatorname{tr}_{f c} \int \frac{d^{4} p}{(2 \pi)^{4}}\left(\frac{1}{\left(p_{0}^{2}-E_{\Delta}^{+2}\right)^{2}}+\frac{1}{\left(p_{0}^{2}-E_{\Delta}^{-2}\right)^{2}}\right), \\
& I_{0}^{\prime}=-2 i \operatorname{tr}_{f c} \int \frac{d^{4} p}{(2 \pi)^{4}} \frac{1}{\left(p_{0}^{2}-E_{\Delta}^{+2}\right)\left(p_{0}^{2}-E_{\Delta}^{-2}\right)} .
\end{aligned}
$$

We write the expressions of these divergent integrals in terms of the temperature and the chemical potential using the Matsubara formulation as

$$
\begin{aligned}
& I_{2}=\operatorname{tr}_{f c} \int \frac{d^{3} p}{(2 \pi)^{3}}\left(\frac{1}{2 E_{\Delta}^{+}}\left(1-2 \tilde{f}\left(E_{\Delta}^{+}\right)\right)+\frac{1}{2 E_{\Delta}^{-}}\left(1-2 \tilde{f}\left(E_{\Delta}^{-}\right)\right)\right) \\
& I_{0}=\operatorname{tr} \int \frac{d^{3} p}{(2 \pi)^{3}}\left[\frac{1}{4 E_{\Delta}^{+3}}\left(1-2 \tilde{f}\left(E_{\Delta}^{+}\right)-2 E_{\Delta}^{+} \beta e^{\beta E_{\Delta}^{+}} \tilde{f}^{2}\left(E_{\Delta}^{+}\right)\right),+\frac{1}{4 E_{\Delta}^{-3}}\left(1-2 \tilde{f}\left(E_{\Delta}^{-}\right)-2 E_{\Delta}^{-} \beta e^{\beta E_{\Delta}^{-}} \tilde{f}^{2}\left(E_{\Delta}^{-}\right)\right)\right] \\
& I_{0}^{\prime}=2 \operatorname{tr}_{f c} \int \frac{d^{3} p}{(2 \pi)^{3}} \frac{1}{4 E_{\Delta}^{-} E_{\Delta}^{+}}\left[\frac{2}{E_{\Delta}^{+}-E_{\Delta}^{-}}\left(f\left(E_{\Delta}^{+}\right)-f\left(E_{\Delta}^{-}\right)\right)+\frac{2}{E_{\Delta}^{+}+E_{\Delta}^{-}}\left(1-f\left(E_{\Delta}^{-}\right)-f\left(E_{\Delta}^{+}\right)\right)\right] .
\end{aligned}
$$

The pion field does not mix with other fields due to its pseudoscalar nature. However, the sigma meson field mixes with diquark and anti-diquark fields, and we have to diagonalize the mass matrix.

The diquark Lagrangian is derived by calculating $d d^{*}$ and $d^{*} d$ terms in a similar way. Where we have to take care that the first order derivative with timelike component is not vanish. The $d d$ and $d^{*} d^{*}$ 
terms describe the diquark mixing. We decompose the diquark baryon fields $d$ and the mean field value $\Delta_{0}$ to $d=\phi_{1}+i \phi_{2}$ and $\Delta_{0}=\Delta_{R}+i \Delta_{I}$ and their conjugates. With these fields, we can write

$$
\begin{aligned}
\mathcal{L}_{D}= & \frac{1}{2}\left(g_{d}^{2} I_{0} \partial^{\mu} \phi_{1}(x) \partial_{\mu} \phi_{1}(x)-m_{d}^{2} \phi_{1}^{2}(x)\right)+\frac{1}{2}\left(g_{d}^{2} I_{0} \partial^{\mu} \phi_{2}(x) \partial_{\mu} \phi_{2}(x)-m_{d}^{2} \phi_{2}^{2}(x)\right) \\
& +\frac{1}{2}\left[4 g_{d}^{2} I_{0} \mu\left(\phi_{2}(x) \partial^{0} \phi_{1}(x)-\phi_{1}(x) \partial^{0} \phi_{2}(x)\right)-2 g_{d}^{2} I_{0} \Delta^{2}\left(\phi_{1}(x) \phi_{2}(x)+\phi_{2}(x) \phi_{1}(x)\right)\right] .
\end{aligned}
$$

The mass of the diquark baryon is written as $m_{d}^{2}=\left(\frac{1}{H_{0}}-2 I_{2}+2 \Delta^{2} I_{0}\right) / I_{0}$.

Sigma meson and diquark baryon have mixing because they are scalar particle. We write $\Delta_{0}$ and $d$ in terms of the real and the imaginary parts

$$
\mathcal{L}_{s d}=-\frac{1}{2} 2 \sqrt{2} \Delta m \sqrt{I_{0} / I_{0}^{\prime}}\left(\phi_{1} s+\phi_{2} s+s \phi_{1}+s \phi_{2}\right)
$$

$\Delta^{2}$ has been defined as $g_{d}^{2}\left|\Delta_{0}\right|^{2}$, and $\Delta=\sqrt{2} g_{d} \Delta_{R, I}$.

\section{Numerical results}

We would like to show numerical results in this section. For this purpose we fix the parameters of the PNJL model following the paper of Brauner et al., the list of which is provided in Table I of the paper [9]. These parameters provide the pion mass in the free space as $m_{\pi}=140 \mathrm{MeV}$ and the pion decay constant at $f_{\pi}=\sqrt{2 / 3} \times 93 \mathrm{MeV}=76 \mathrm{MeV}$. The coupling constants taken are $G=H=7.23$ $\mathrm{GeV}^{-2}$ and the cut-off momentum $\Lambda=657 \mathrm{MeV}$.

\subsection{Phase diagram and order parameters}

We start showing the chiral condensate, the diquark condensate and the Polyakov loop with and without the coupling of the quark with the Polyakov loop. We show in Figs. 1 and 2 the behavior of the condensates as functions of temperature at zero chemical potential $(\mu=0)$. The critical temperature of

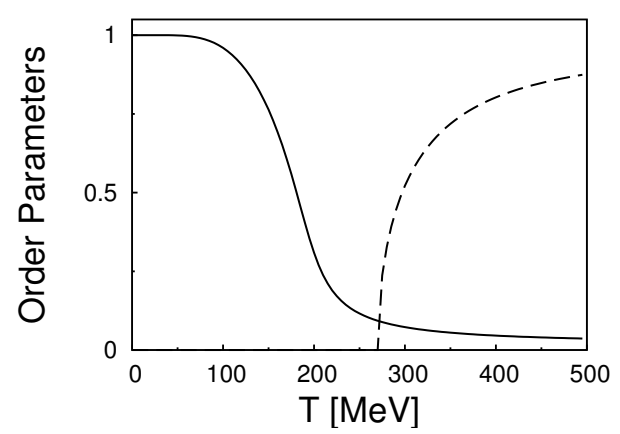

Fig. 1. The chiral condensate $\sigma$ in unit of $\sigma_{0}$ (solid curve) and the Polyakov loop $\Phi$ (dashed curve) as functions of temperature at zero chemical potential $(\mu=0)$ for the case of the NJL model.

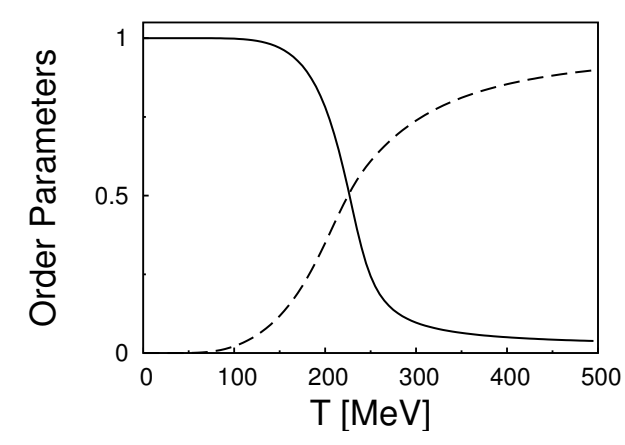

Fig. 2. The chiral condensate $\sigma$ (solid curve) and the Polyakov loop $\Phi$ (dashed curve) as functions of temperature at zero chemical potential $(\mu=0)$ for the case of the PNJL model.

the Polyakov loop is set at $T_{0}=270 \mathrm{MeV}$ for the case without the quark and Polyakov loop coupling. When the coupling between the quark and the Polyakov loop is introduced in the PNJL model, the two critical temperatures are strongly correlated as shown in Fig. 2. We show then the case at finite chemical potential $(\mu=100 \mathrm{MeV})$ in Figs. 3 and 4 . In this case, the diquark condensate becomes finite and makes the chiral condensate smaller where the diquark condensate is finite. Again the coupling of the Polyakov loop makes the de-confinement transition smooth and both the critical temperatures become quite similar. 


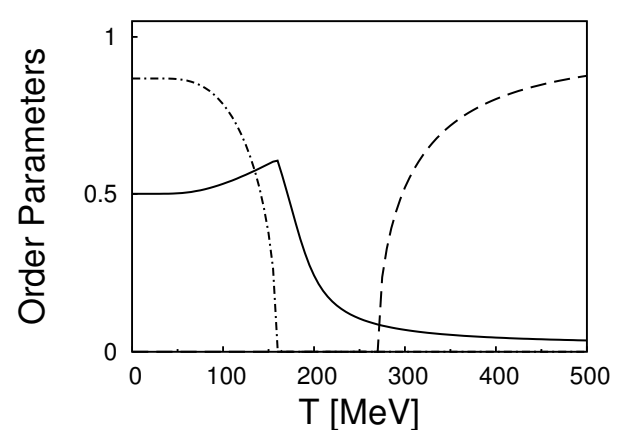

Fig. 3. The chiral condensate $\sigma$ (solid curve), the diquark condensate $\Delta$ (dash-dotted curve) in unit of $\sigma_{0}$ and the Polyakov loop $\Phi$ (dashed curve) as functions of temperature at finite chemical potential $(\mu=100 \mathrm{MeV})$ for the case of the NJL model.

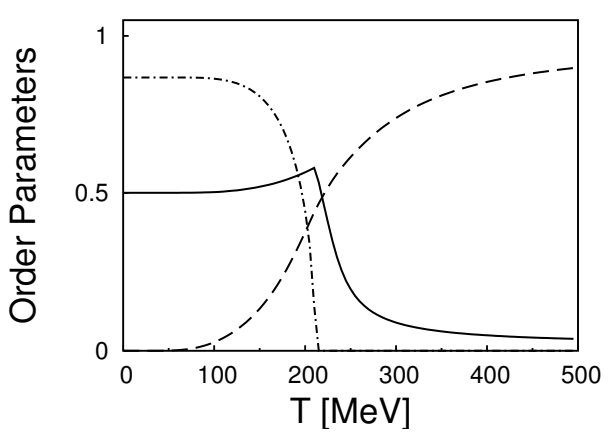

Fig. 4. The chiral condensate $\sigma$ (solid curve), the diquark condensate $\Delta$ (dash-dotted curve) in unit of $\sigma_{0}$ and the Polyakov loop $\Phi$ (dashed curve) as functions of temperature at finite chemical potential $(\mu=100 \mathrm{MeV})$ for the case of the PNJL model.

\subsection{Meson and diquark masses}

We show the behaviors of sigma and pion masses as functions of temperature with and without the quark Polyakov loop coupling at zero chemical potential $(\mu=0)$ in Figs. 5 and 6 . Due to the small explicit chiral symmetry breaking $m_{0}=5.4 \mathrm{MeV}$, the pion as a Nambu-Goldstone boson acquires a small mass of $140 \mathrm{MeV}$ at zero temperature, while the sigma mass stays around twice of the constituent quark mass $(m=305 \mathrm{MeV})$. As the temperature increases the pion mass start to increase, while the sigma mass decreases until the temperature approaches the crossover temperature of $180 \mathrm{MeV}$. After the two masses meat at the crossover temperature, they increase together as shown in Fig. 5.

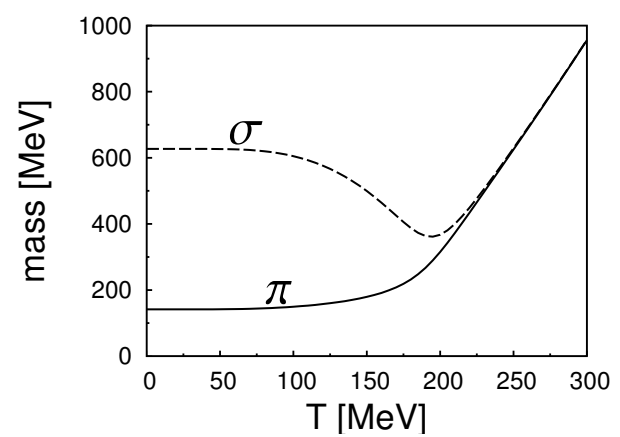

Fig. 5. Sigma (dashed curve) and pion masses (solid curve) in $\mathrm{MeV}$ as functions of temperature in $\mathrm{MeV}$ at zero chemical potential $(\mu=0)$ for the case of the NJL model.

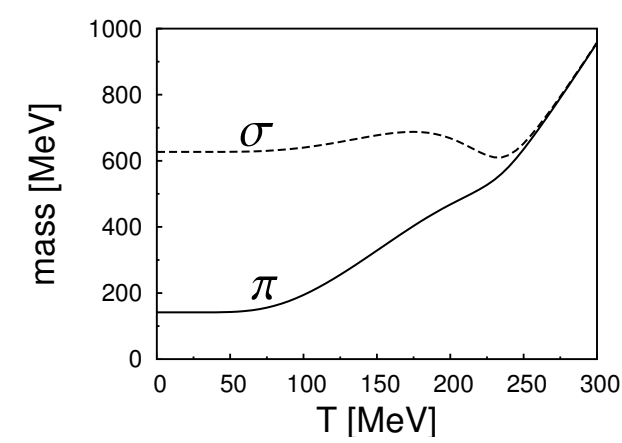

Fig. 6. Sigma (dashed curve) and pion masses (solid curve) in $\mathrm{MeV}$ as functions of temperature at zero chemical potential $(\mu=0)$ for the case of the PNJL model.

When the quark Polyakov loop coupling is introduced, the increase of the pion mass becomes earlier and slower with temperature as shown in Fig. 6 due to the fact that the quark mass drops slower in larger temperature range as shown in Fig. 2. The sigma mass does not drop much but only at the crossover temperature and then both the masses increase together with the temperature at the crossover temperature of $T_{\chi}=225 \mathrm{MeV}$.

We show now the behaviors of the meson masses and the diquark masses as functions of the chemical potential $\mu$ in Figs. 7 and 8. Until the onset of the diquark condensate, the pion mass stays at $140 \mathrm{MeV}$ and the sigma mass very slightly increases due to the renormalization constants. On the other 


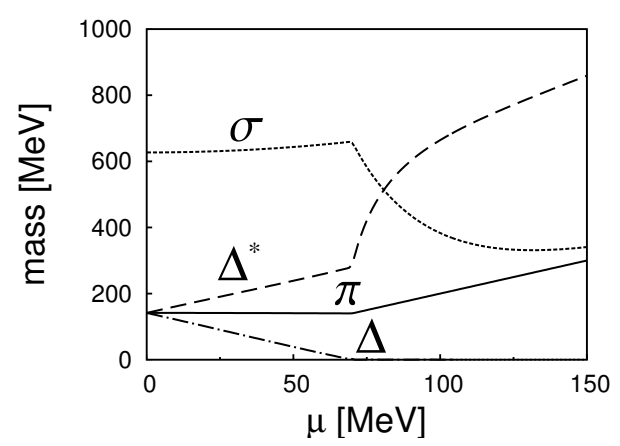

Fig. 7. The sigma (dotted curve), the pion masses (solid curve) and diquark masses (dash-dotted and dashed curves) in $\mathrm{MeV}$ as functions of the chemical potential $\mu$ at zero temperature $(\mathrm{T}=0)$. The sigma and diquark coupling is dropped when $\Delta \neq 0$.

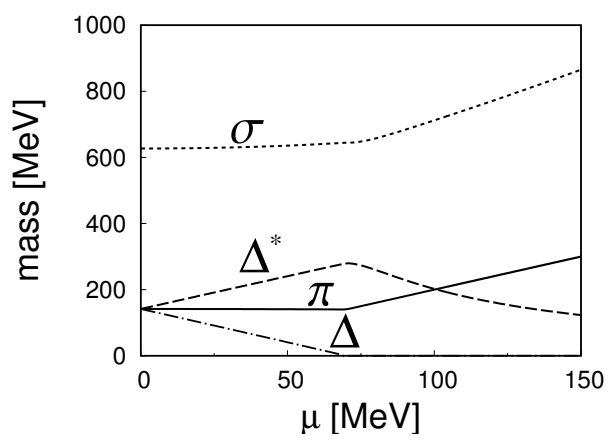

Fig. 8. The sigma (dotted curve), the pion masses (solid curve) and diquark masses (dash-dotted curve) and (dashed curve) in $\mathrm{MeV}$ as functions of the chemical potential $\mu$ at zero temperature $(\mathrm{T}=0)$. The sigma and diquark coupling is introduced when $\Delta \neq 0$.

hand the diquark masses are the same as the pion mass at $\mu=0$ due to the Pauli-Güersey symmetry. One of the diquarks decreases linearly and the other increases linearly with $\mu$. This is due to the trivial fact that there exist diquark and anti-diquark states at $M_{d}= \pm 140 \mathrm{MeV}$ and with the chemical potential the excitation energy of these states from the chemical potential changes linearly with $\mu_{B}=2 \mu$.

After diquark condensation, which occurs at $\mu=\frac{1}{2} m_{\pi}$, the pion mass increases linearly with $\mu$, because the energy normalization should be made at the positive energy diquark mass. This energy normalization corresponds to the fact that one of the diquark state stays at the zero excitation energy as shown in Figs. 7 and 8. As for the sigma and the other diquark mass, they couple each other after diqaurk condensation $\Delta \neq 0$. In Fig. 7 , the coupling is suppressed and hence the sigma mass drops slightly as $\mu$ approaches the crossover chemical potential $\mu \sim 150 \mathrm{MeV}$ and increases together with the pion mass. The diquark mass increases rapidly with the chemical potential after diquark condensation. On the other hand, when the coupling between the sigma meson and the diquark-baryon is introduced, the behaviors of those masses change largely as shown in Fig. 8. The coupling of the sigma meson and the diquark baryon is very large.

\subsection{Equation of state of quark-hadron matter}

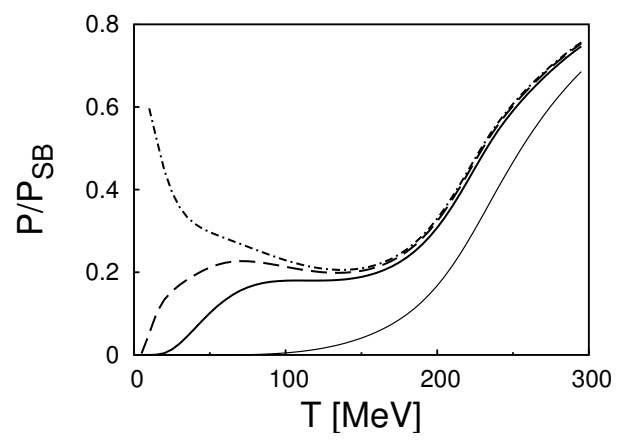

Fig. 9. The pressures divided by the Stefan-Boltzmann pressure for various chemical potentials $(\mu=0,40$ and $47 \mathrm{MeV}$ ) below diquark condensation as functions of temperature. Shown also is the one with only the quark degree. 


\section{EPJ Web of Conferences}

We study the equation of state (EOS) of quark-hadron matter with and without the hadron degrees of freedom at various chemical potential as functions of the temperature $T$ in Figs. 9. The pressure divided by the Stefan-Boltzmann pressure is plotted in these figures. The thin solid curve denotes the EOS with only the quark degree of freedom. The pressure stays almost zero before de-confinement transition and increases suddenly after de-confinement transition with temperature. The other curves include the contribution of hadrons and hence the EOS depends on the chemical potential. Shown are the EOS's at $\mu=40 \mathrm{MeV}$ and $\mu=47 \mathrm{MeV}$. Because of the fact that the pion mass and the diquark masses are small, the contributions of these degrees of freedom make the EOS largely different from that of the quark degree only. The EOS closer to the critical temperature of the onset of diquark condensation $\mu=70 \mathrm{MeV}$ behaves singularly as it approaches the zero temperature indicating closeness to the critical point.

\section{Conclusion}

We have studied the quark-hadron matter at finite temperature and density by using the NJL model with the Polyakov loop potential. We consider the case of two color and therefore the diquark baryon is boson. We work out the thermodynamical potential with the order parameters of the Polyakov loop, the chiral condensate and the diquark condensate. We have worked out the hadron properties in all the temperature and density plain.

The Polyakov loop plays important role at finite temperature and the de-confinement phase transition is now correlated with chiral condensation. The temperature where those values become half values of the maximum values, both the temperatures agree at $T_{\chi}=T_{d e c}=225 \mathrm{MeV}$. In the finite chemical potential case at small temperature $T<T_{d e c}$, the diquark condensate plays important role. The diquark condensate becomes finite at $\mu=\frac{1}{2} m_{\pi}$. This is simply the Bose-Einstein condensation. From this chemical potential the diquark condensate grows quickly and stay finite at large chemical potential. As the diquark condensate sets in, the chiral condensate decreases.

The meson and diquark masses behave naturally according to chiral and diquark condensation. The pion mass stays constant until diquark condensation and grows linearly with the chemical potential after diquark condensation. The sigma meson mass is about twice of the quark mass until diquark condensation. Due to the coupling of the sigma meson with diquark baryons, the mixed hadrons of sigma and diquark behave quite differently from the case without the coupling after diquark condensation. One of the diquark stays zero after diquark condensation.

The EOS at various chemical potential shows an interesting behavior, when we include the hadrons as the thermal degrees of freedom. Below diquark condensation, when the chemical potential approaches the critical chemical potential $\mu \sim \frac{1}{2} m_{\pi}$, the pressure starts to diverge at zero temperature. This is a signal of phase transition. In fact, the diquark mode stays at $m_{d}=m_{\pi}$ and when the quark chemical potential becomes a half of the pion mass $\mu=\frac{1}{2} m_{\pi}$, diquark condensation as the BoseEinstein condensation takes place.

\section{References}

1. Y. Nambu and Jona-Lasinio, Phys. Rev. (1961) 345.

2. K. Fukushima, Phys. Rev. D68 (2003) 045004.

3. K. Fukushima, Phys. Lett. B591 (2004) 277.

4. C. Ratti, M.A. Thaler and W. Weise, Phys. Rev. D73 (2006) 014019.

5. S. Hands, J. Montvay, L. Scorzato, J. Skullerud, Eur. Phys. J. C22 (2001) 451.

6. S. Hands, S. Kim, J.-I.Skullerud, Eur. Phys. J C48 (2006) 193.

7. Kogut, Toublan, Sinclair, Nucl. Phys. B642 (2002).

8. C. Ratti and W. Weise, Phys. Rev D70 (2004) 054013.

9. Brauner, Fukushima, Hidaka, Phys. Rev D80 (2009) 074035.

10. T. Eguchi, Phys. Rev. D10 (1976) 2755.

11. Huang, Zhuang, Chao, Phys. Rev. D65 (2002) 076012. 